\title{
Estimation of the Fracture Probability Parameters for Specimens Made by JIS SM490A Steel
}

\author{
Rafael Magalhães de Melo Freire ${ }^{a}$ (1), Shuji Aihara ${ }^{b}$, Armando Hideki Shinohara ${ }^{a *}$ (), Shuhei \\ Yoshizu ${ }^{b}$, Petrônio Barros Mesquita ${ }^{a}$
}

${ }^{a}$ Universidade Federal de Pernambuco, Departamento de Engenharia Mecânica, Recife, PE, Brasil.

${ }^{b}$ The University of Tokyo, 7-3-1 Hongo, Bunkyo, Tokyo, Japan.

Received: October 22, 2020; Revised: March 24, 2021; Accepted: May 11, 2021

\begin{abstract}
This work established the fracture probability equation for JIS SM490A steel using the computational model supported by the local approach and the standard Crack Tip Opening Displacement (CTOD) fracture toughness experiments. As the experiments were conducted at a low temperature providing conditions for the brittle fracture, the specimen suffers a small plastic deformation until its failure. The computational model tries to represent the experiment results, estimating the scale and shape parameters of the Weibull distribution for the cumulative fracture probability equation. A procedure based on the local approach was executed to find the optimum values for the fracture probability equation. In addition, a plot was provided to show the distribution of likelihood values according to assumed values for the shape and scale parameters. In the end, a comparison between the experimental and predicted statistical distribution of critical CTOD was done successfully, indicating that material toughness can be predicted by the present model.
\end{abstract}

Keywords: Toughness scatter, brittle fracture, Weibull distribution, fracture probability.

\section{Introduction}

Pressurized vessels, boilers, pipes, and structures made by steel under specific environmental conditions can generate catastrophic structural failures when they are subjected to low temperature, suffering a small plastic deformation if the fracture toughness of the steel is very low, especially at welds. Identical mechanical components can also fail when they are submitted to different applied stresses in different environmental conditions. The macro and micro defects have an impact on the material resistance; hence they need to be investigated to avoid catastrophic structural failures. In the steel microstructure, there are micro defects that can cause variation of the material property values, raising the necessity to evaluate it using a statistical tool. The material behavior studies conducted on the cleavage fracture conditions investigating the toughness scatter play an important role in structural integrity.

As explained by Beremin et al. ${ }^{1}$, macro defects present on the mechanical structures independently of material type need to be evaluated using approaches that can support simple and complex cases. Due to the need to improve the performance of structures and to ensure safety in general, studies to predict material behavior are essential. The fracture mechanics study the mechanical processes that lead to the crack propagation and other "defects" and that causes the decreasing of the material resistance and consequently result in its fracture.

A macroscopic defect can decrease the material performance and motivate a failure of a mechanical part. For this reason, pressure vessels, boilers, pipes, and other

*e-mail: shinohara@ufpe.br equipment are periodically submitted to inspections in the industry. However, the inherent presence of microscopic defects induces a variation in the toughness values which can also contribute to the material failure before the expected stress. About the importance of microstructure and microscopic defects, it is important to mention that the thermo-mechanical process can be performed to improve fracture toughness, as well as the cleavage fracture behavior. Using warm pre-stressed material, which is a procedure that includes a previous loading at high temperature, Bordet et al. ${ }^{2}$ found an enhancement of the fracture toughness in comparison with the virgin material. The warm pre-stress and the conservative principle studied in Bordet et al. ${ }^{2}$ had a positive effect on the structural integrity of several types of equipment, such as a nuclear pressure vessel.

There is a natural dispersion of the toughness of the material that occurs depending on the position of the particle that triggers the failure. Anderson ${ }^{3}$ explains that depending on the distance of this particle from the crack, the toughness of the test piece will change. This is because if this particle is closer to the crack tip the energy required will be less compared to a case of the part having this particle at a greater distance from the crack tip. It is worth saying that a large scatter is often revealed on cleavage fracture. Beremin et al. ${ }^{1}$ examined the toughness scatter and presented the statistical model to analyze the cleavage fracture. The toughness scatter studies in mechanical failure were introduced by the weakest link probabilistic theory to obtain the material fracture toughness. Based on this methodology, Ruggieri et al. ${ }^{4}$, Tagawa et al. ${ }^{5}$, and Kawata et al. ${ }^{6}$ investigated the fracture toughness 
distribution obtained from the CTOD test. In Lin et al. ${ }^{7}$, predictions for the fracture toughness are shown, taking into account the microstructure and temperature, using a quantitative weakest-link model. These results can infer that there is a variation of the fracture toughness values for a fixed or variant temperature.

Temperature and loading conditions are also factors to be considered in the fracture behavior of ferritic steels, with low alloy, or high alloy grades. When a mechanical part is subjected to a load higher than the yield and rupture points at a temperature that does not affect the material's behavior, a ductile fracture can occur with the part since it is probably working in the stability zone. However, if a high load is applied to this mechanical part at a lower temperature, its fracture can become unstable by cleavage which may cause unexpected failure and accidents. Lin et al. ${ }^{7}$ predicted the variation of the fracture toughness value in function of the temperature when showing that this value decreases according to the temperature reduction, which means the material can be embrittled depending on the working temperature. There is a temperature range that sets the material in the ductile-tobrittle transition causing difficulties to predict the toughness, consequently scattering of its values.

Studies have been conducted by many researchers through experiments that use the standard three-point bend specimen in the ductile-to-brittle transition or brittle region to investigate constraint effects on the fracture toughness ${ }^{8}$. As explained by Ruggieri ${ }^{9}$, the geometry of the specimen, which has a high ratio of crack depth and specimen height (a/W $\geq 0.5$ ), can contribute to the stress triaxiality ahead of the crack front, as well as to the cleavage fracture conditions limiting the plasticity scale.

Due to the variation in the cleavage fracture toughness data and the weakest link trigger, it is important to use a statistical model to assess the cleavage fracture as conducted by Beremin et al. ${ }^{1}$. Studies based on fracture probability have been widely applied with the Weibull distribution. Sih ${ }^{10}$ explains that Weibull initiated this work which was characterized as a statistical function that is an important tool to evaluate the failure probability of a material submitted to macro defects. Kunigita et al. ${ }^{11}$ mentioned that the fracture toughness can be successfully explained by the stochastic behavior of the toughness, which can be shown through the Weibull stress distributions. In order to provide a computational model that predicts the steel toughness taking into account the microstructure influence, it is necessary to use the data from multiple specimens to calibrate the parameters of the critical Weibull stress distributions as quoted in Kunigita et al. ${ }^{11}$.

Through a probabilistic model that takes into account the effects of plastic strain on cleavage fracture, Ruggieri ${ }^{9}$ demonstrates that the fracture toughness values can attain geometry independence with the application of the modified Weibull stress methodology.

Gao et al. ${ }^{12}$ propose a new calibration manner to find the parameters for the Weibull distribution using the toughness values measured under specific experimental conditions. Thereby, a strong sensitivity with the Weibull shape parameter was revealed by this new procedure, as well as the necessary micromechanical values to assess flawed structural components.
The fracture mechanics tests conducted in laboratories provide some material properties which are relevant information for describing a mechanical part fracture resistance when it contains a crack. The fracture toughness, which is a parameter that can be quantified, plays an important role to characterize the material, evaluate the performance, and assure the material quality used in many kinds of structures.

There are many parameters related to fracture mechanics studies, such as the stress intensity factor $(\mathrm{K})$, the crack-tip opening displacement (CTOD), the elastic energy release rate $(\mathrm{G})$, the J-integral, and the crack-tip opening angle (CTOA). However, this work concentrates on the standard CTOD test dealing with CTOD values and fracture probability to portray the toughness scatter.

The target of this work is to represent the dispersion of the tested material toughness obtained by CTOD tests performed in the laboratory through a computational model based on a local approach. The Weibull statistical equation is employed to describe the fracture toughness scatter and the distribution of critical CTOD of the experiments. The present model was executed to predict the cumulative failure probability equation finding optimum values for its parameters, which are the scale and shape parameters related to the Weibull distribution equation. Through the developed procedure, it was possible to derive the Weibull parameters that portray the CTOD laboratory test results.

\section{Analysis Methods}

\subsection{Local approach}

It is assumed that the fracture initiation point occurs close to the discontinuity and the stress distribution behaves in a non-linear manner depending on the distance from the crack tip. Figure 1 shows the stress distribution considering

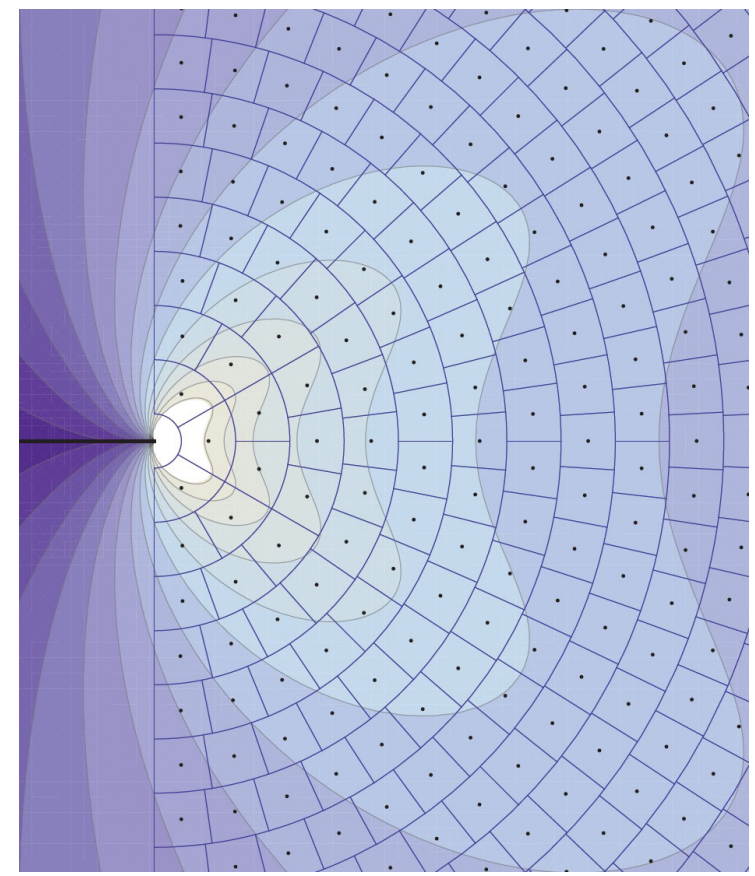

Figure 1. Elements and stress pattern near the crack tip. 
a view of the element arrangement near the crack tip. It is worth mentioning that these elements are not developed by the finite-element analysis, they were right generated using a computational model to represent a material unit for calculating the probability of fracture with the weakest-link theory. Furthermore, the computational model, which represents the present model, adopted this element arrangement to predict fracture location near the crack tip, considering the maximum principal stress for each element.

A volume element subjected to cleavage fracture conditions fails when the maximum principal stress acting on the volume element $\left(\sigma_{1}\right)$ is higher than the fracture stress $\left(\sigma_{f}\right)$ in a characteristic distance from the crack tip. Lin et al. ${ }^{7}$ evidence that the crack starts with the highest probability at a critical radial distance from the crack tip. However, the exact point of fracture initiation is a matter to be addressed by statistical analysis, because it tends to be very scattered.

Differing from the type of fracture that initiates at many sites due to the micro-void growth and coalescence, the cleavage fracture abruptly happens when the fracture initiation condition is reached in the failure of the first volume element resulting in crack propagation across the specimen or the structural member. That is the weakest-link mechanism in which the nucleation takes place and generates an unstable propagation of a critical flaw of a part or structure subjected to a load.

The cleavage fracture can be controlled by the dimensions of the flaws and by the stress condition, although the random nature of the micro defects affects the scatter of fracture stress and fracture toughness values. Considering this phenomenon, Beremin et al. ${ }^{1}$ introduced the local approach, which tries to explain the scatter of fracture toughness based on the weakest-link mechanism. Based on the local approach, the probabilistic model of fracture initiation helps to describe this scattering.

This model is explained by Beremin et al. ${ }^{1}$ also utilized the Weibull distribution for describing fracture toughness scatter. The Weibull theory is a local criterion that takes account of plastic strain. The scattering in the experimental results can explain the fracture toughness variation of the material. It is assumed that the fracture material behavior obeys a statistical distribution of stress through the Weibull distribution, as shown in Equation 1.

$$
P\left(\sigma_{f}\right)=1-e^{-\left(\frac{\sigma_{f}}{\beta_{f}}\right)^{m}}
$$

$P\left(\sigma_{f}\right)$ represents the statistical distribution of the fracture stress, $\sigma_{f}$, while $m$ is the Weibull shape parameter, and $\beta_{f}$ is the Weibull scale parameter. The Weibull distribution shown by Equation 1 is used to represent the statistical variation of the fracture stress, in order to predict the toughness scattering.

Considering Equation 1, the probability of specimen fracture can be expressed based on the weakest link assumption, as shown in Equation 2:

$$
P_{\text {specimen }}=1-\prod_{i=1}^{N}\left(1-P\left(\sigma_{f}<\sigma_{1 i}\right)\right)
$$

Where $\sigma_{1 i}$ is maximum principal stress acting on the $i$-th volume element, which is determined by the HRR (HutchinsonRice-Rogengren non-linear crack-tip stress-strain analysis), as shown by Rice and Rosengren ${ }^{13}$ and Hutchinson ${ }^{14} . N$ is the total number of the volume elements.

It is generally accepted that cleavage crack initiation in steels is triggered by microcrack nucleation from hard second phase particles. The microcrack nucleation is controlled by strain, and linear dependence of the probability of microcrack nucleation on applied strain was found by Gurland ${ }^{15}$. Since Equation 2 does not ponder the strain effect, the stress-strain criterion was applied to evaluate it. Equation 3, which incorporates the strain effect, takes into account the linear dependence of the probability of microcrack nucleation.

This present work incorporates in Equation 2 the effect of strain on crack initiation as considered by Bordet et al. ${ }^{2}$, which also uses a statistical fracture model based on the Weibull stress criterion. Through Equation 3, it is assumed that the microcrack formation is led by a compound of stress and strain, which influences the probability of fracture of a volume element.

$P_{\text {element }}=P\left(\sigma_{f}<\sigma_{1}\right) P\left(d \varepsilon_{p}\right)$

The term $P\left(d \varepsilon_{p}\right)$ represents the microcrack nucleation probability in a volume element during a time step increment. The studies of Gurland ${ }^{15}$ was used to assume it as a linear expression, $P\left(d \varepsilon_{p}\right)=1.0 d \varepsilon_{p}$, where $d \varepsilon_{p}$ is the plastic strain increment during the time step and it can be calculated from the HRR solution ${ }^{13,14}$. Hence, the specimen fracture probability can be alternatively expressed by Equation 4:

$$
P_{\text {specimen }}=1-\prod_{i=1}^{N}\left(1-P\left(\sigma_{f}<\sigma_{1}\right) P\left(d \varepsilon_{p}\right)\right)
$$

It is possible to calculate the specimen fracture probability, $P_{\text {specimen }}$ through Equations 2 and 4, which are expressions for the stress-only criterion and the stress-strain criterion, respectively.

In the CTOD test, each volume element near the specimen's crack tip is exposed to the stress, $\sigma_{1 i}$, and the set of these elements can be related to a parameter. The Weibull stress, $\sigma_{W}$, is calculated through a sum of each stress, taking into account the parameter $\mathrm{m}$, as shown in Equation 5. It can be used as a probability fracture parameter for Weibull stress distribution.

$\sigma_{W}=\left\{\sum_{i=1}^{N} \sigma_{1 i}^{m}\right\}^{1 / m}$

It is necessary to assume values to $\mathrm{m}$ in order to calculate the Weibull stress, $\sigma_{W}$. If a number of fracture toughness values are obtained by an experiment, values of $\mathrm{m}$ and $\beta_{f}$ can be determined, using Equation 5 by, e.g., the mostlikelihood method.

The probability of specimen fracture, $P_{\text {specimen }}$, can be calculated as a function of $\delta$, assuming values for $\left\{m, \beta_{f}\right\}$, although, critical CTOD values, $\delta_{c}$, were obtained through the experiments. Then, Equation 6 can be used as the likelihood function: 
$L=\log \prod_{n=1}^{M} P\left(\delta_{c(n)}\right)$

Focusing on the maximum values for likelihood, it is possible to plot the values of $\mathrm{L}$ in the 2-dimensional graphic as a function of $\left\{m, \beta_{f}\right\}$, hence the optimum values of $\left\{m, \beta_{f}\right\}$ can be found.

It is worth mentioning that, the statistical distribution of critical CTOD can be written as Equation 7, including CTOD parameters $m_{\delta}$ and $b_{\delta}$, which was also used in the studies of Ruggieri et al. ${ }^{4}$.

$P\left(\delta_{c}\right)=1-e^{\left(-\frac{\delta_{c}}{b_{\delta}}\right)^{m_{\delta}}}$

The fracture data acquired from experimental testing can be analyzed using Weibull statistics. Despite the initial crack length and specimen width can be changed depending on the sample, the Weibull shape parameter $m_{\delta}$ is near 2, the theoretical value for cleavage fracture, as mentioned by Ruggieri et al. The shape parameter and the Weibull scale parameter of the computational model can be calibrated using the experimental data explained previously from the fracture probability equation. Considering the computational and experimental results, a Weibull distribution plot can be provided in a logarithmic graphic, in order to compare their failure probability equations.

\subsection{Present model}

The Hutchinson-Rice-Rosengren (HRR) proposed a method to portray the non-linear stress-strain distribution near a crack-tip, and its equation is known as the HRR singularity which assumes form for plane stress and plane strain ${ }^{3,13,14}$. Since this work uses Equation 4 instead of Equation 5, a method can be applied to express the stress distribution for each element near the crack-tip. HRR singularity or even the finite element method, FEM, could be also employed to obtain it. However, HRR singularity is considered in this work for simplicity. Hence the maximum principal stress acting on a volume element $\left(\sigma_{1}\right)$ is expressed by Equation 8:

$$
\sigma_{i j}(r, \theta)=\sigma_{0}\left(\frac{E J}{\alpha \sigma_{0}^{2} I r}\right)^{\frac{1}{n+1}} \tilde{\sigma}_{i j}(\theta)
$$

Equation 8 is an expression for a plane strain condition in planar-polar coordinates centered and aligned ahead in the crack-tip. It uses the yield stress $\left(\sigma_{0}\right)$, Young's modulus (E), a strain hardening exponent $(n)$, and a dimensionless constant $(\alpha)$. The constant expressed as $I$ is related to the value of $n$, and the dimensionless function, $\tilde{\sigma}_{i j}$, is dependent on $n$ and $\theta$.

For the calculation procedure, the tensile behavior is represented by the temperature which was considered the same for the tested material $\left(-130^{\circ} \mathrm{C}\right)$, therefore $\sigma_{0}$ and $n$ are assumed equal to $500 \mathrm{MPa}$ and 5 ; respectively. The crack-tip opening displacement (CTOD), $\delta$, is used to calculate $J$ integral through Equation 9, in order to use it for the maximum principal stress calculation for each element.
In Equation 9, the factor 2 that multiplies $\sigma_{0} \delta$ was applied since it accounts for strain-hardening, while factor 1 is used for the Dugdale's strip-yield model.

$$
J=2 \sigma_{0} \delta
$$

The present model uses Equations 8 and 9, considering the critical CTOD values from the experiments, that are numbered from $j=1$ to $k$. Then, the probability of specimen failure, $P_{\text {specimen }}$, can be calculated considering each $J_{c}$, which is $J$ integral related to $\delta_{c(j)}(j=1 \sim k)$, and the maximum principal stress, $\sigma_{1(i)}(i=1 \sim N)$, for each element. It is worth mentioning that the small distance from crack-tip, $r<\delta$, was neglected for the $P_{\text {specimen }}$ calculation through Equations 2 and 4 because the stress triaxiality is low in this region.

It is known that the toughness tends to be scattered due to the applied condition for the test that places the material in the brittle fracture zone, although the computational model can depict the toughness scatter in this zone through a predicted failure probability equation, estimating its parameters and evaluating them as executed by Minami et al. ${ }^{16}$. There are values of scale and shape parameters for the failure probability that can represent the material behavior in the CTOD testing. For this reason, a procedure is carried out to verify the optimum parameters for the equation based on the Weibull distribution of critical CTOD. Figure 2 shows a flow chart that explains the procedure to calculate the likelihood function and to plot those values for multiple assumed parameters. Equation 10 represents the likelihood function through the probability density function, which is calculated from the critical CTOD, $\delta_{c}$, and the probability of specimen fracture, $P_{\text {specimen }}$. To obtain those functions, the cumulative Weibull distribution function, $C D F_{W}$, have to be assembled assuming that the critical CTOD obeys the Weibull statistical distribution, compound of shape and scale parameters, $m_{\delta c}$ and $\beta_{\delta c}$.

$$
L=\log \left\{\prod_{j=1}^{k} P D F_{N}\left(\delta_{c W(j)} ; \mu_{N(j)}, \sigma_{N}\right)\right\}
$$

Regarding the region of the CTOD specimen contemplated on the computational calculation, it was admitted that each volume element was scaled as $0.02 \mathrm{~mm}$ and aligned at $r \leq 2.0 \mathrm{~mm}$ from the crack-tip. In the specimen thickness direction, 500 volume elements were aligned every $10 \mathrm{~mm}$ along this direction. The calculation procedure was applied taking into account the same element distribution and size for the stress-only criterion and stress-strain criterion.

Making a summary of it, the proposed model uses the stress only criterion model on a computational simulation combining the probability Equations 1 and 2, which respectively represent the statistical distribution of local fracture stress and the weakest-link probability of fracture, and Equations 8 and 9, which express the stress distribution near crack-tip. Furthermore, the stress-strain criterion is applied through Equations 1 and 4, while the Weibull stress criterion through Equation 5 is used for comparison. 


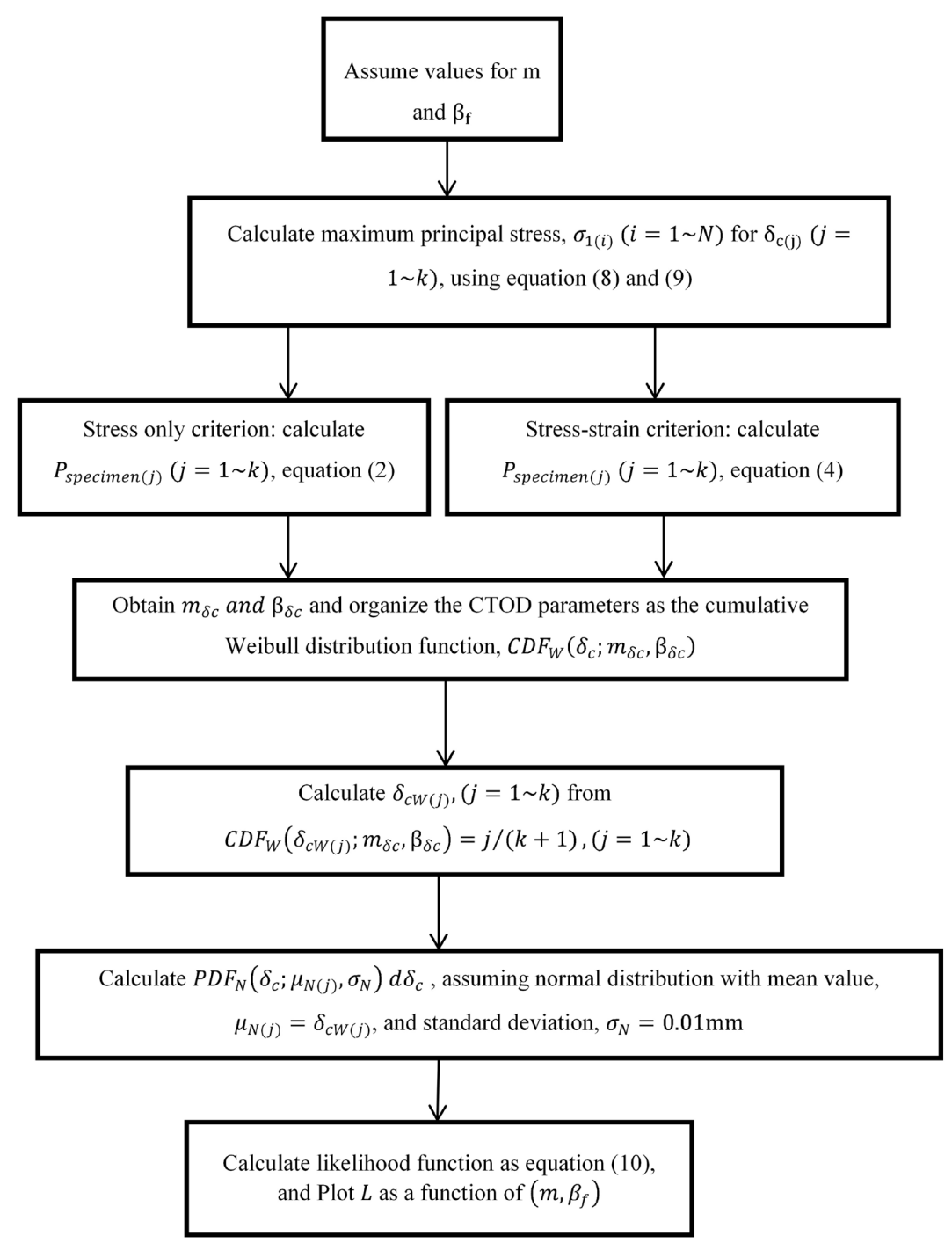

Figure 2. Flow chart of the procedure for likelihood function plot.

\subsection{CTOD Testing}

The tests were carried out in the laboratory using the specimens of JIS SM490A steel with dimensions according to WES $1108-1995^{17}$ testing procedure by the Japanese Welding Engineering Society (JWES). The laboratory and machine where the tests are carried out are shown in Figures 3. In Figure 4, the geometry of the samples and the fatigue pre-crack which was introduced in the specimens are shown. The material, properties, and chemical analysis of the specimen were the grade JIS SM490A ferrite-pearlite steel, as shown in Table 1.

The three-point bending tests were executed for 38 samples which provided the data independently of each other. The data that was collected from the test was later used for critical CTOD calculations and to make computational simulations of the sample behavior through the Mathematica Wolfram.
Table 1. Steel chemical composition and mechanical properties.

\begin{tabular}{cccccc}
\hline \multicolumn{6}{c}{ Chemical composition (weight\%) } \\
\hline $\mathrm{C}$ & $\mathrm{Si}$ & $\mathrm{Mn}$ & $\mathrm{P}$ & $\mathrm{S}$ & $\mathrm{V}$ \\
\hline 0.15 & 0.36 & 1.35 & 0.015 & 0.005 & 0.034 \\
\hline \multicolumn{5}{c}{ Mechanical properties } \\
\hline Yield Point & Tensile Strength & Elongation (\%) \\
\hline $415 \mathrm{MPa}$ & \multicolumn{2}{c}{$525 \mathrm{MPa}$} & \multicolumn{2}{c}{30} \\
\hline
\end{tabular}

After performing the bending tests with all CTOD samples, it was possible to obtain all the data of the thirty-eight specimens: the load applied during the tests and their respective values of crack tip opening displacement. The experimental parameter was obtained using the data extracted from the experimental tests and from the specimen analysis; this step is exemplified by Figures 5 and 6. 


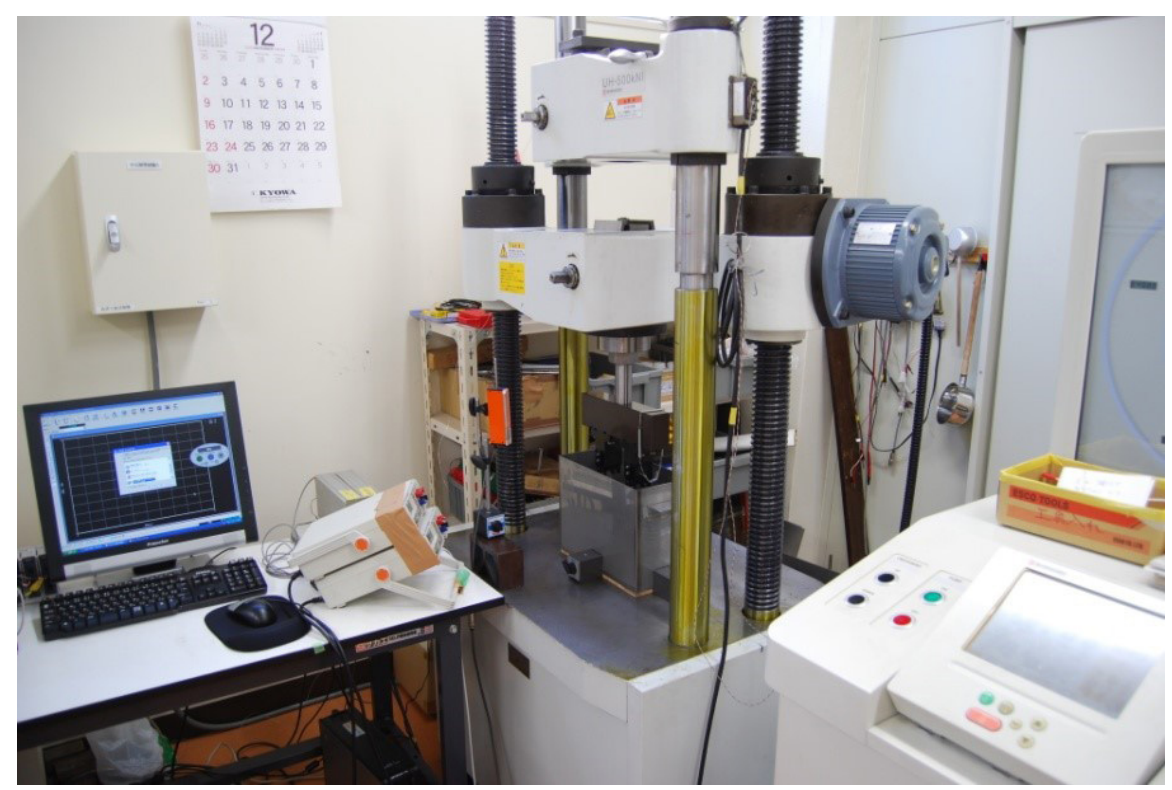

Figure 3. CTOD testing environment.
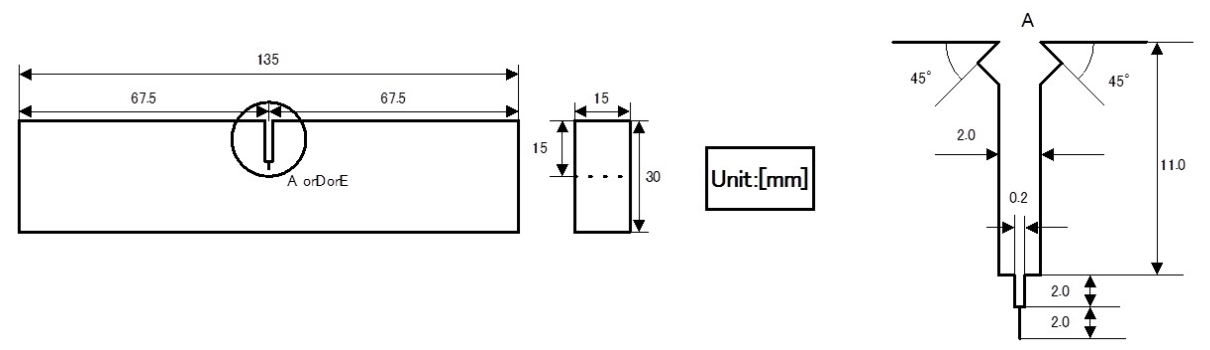

Figure 4. Geometry of the specimen.
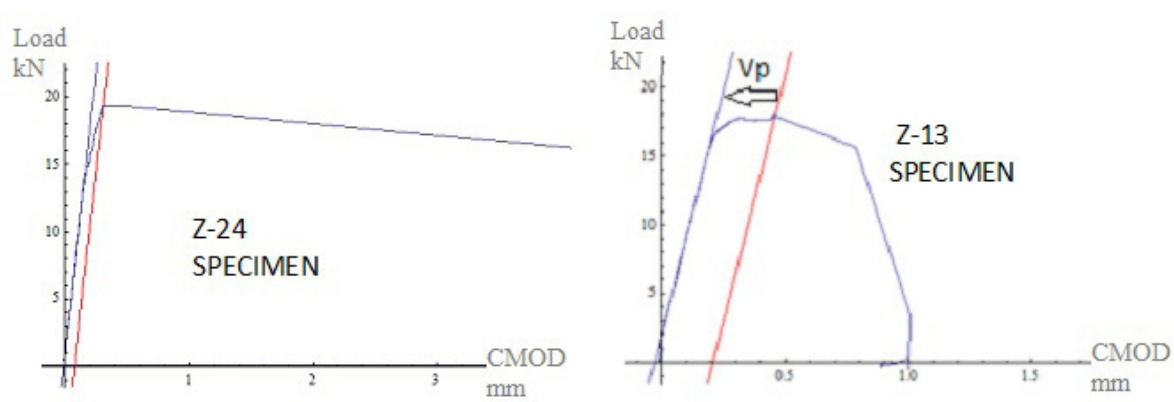

Figure 5. Material behavior graphics (Load x Crack Mouth Opening Displacement).

$\delta=\frac{K^{2}\left(1-v^{2}\right)}{2 \sigma_{Y} E}+\frac{r_{p}\left(W-a_{0}\right) V_{p}}{r_{p}\left(W-a_{0}\right)+a_{0}+z}$

Then, the CTOD values are calculated for each sample based on the Equation 11 presented in WES1108-1995 ${ }^{17}$, and using the data extracted from the experimental tests. It can be seen that Equation 11 uses the crack mouth opening displacement $\left(V_{p}\right)$, stress intensity factor $(K)$, Poisson's ratio $(v)$, yield stress $\left(\sigma_{Y}\right)$, Young's modulus $(E)$, specimen width $(W)$, crack length $\left(a_{0}\right)$, knife-edge height $(z)$, and rotational factor $\left(r_{p}=0.4\right)$
Table 2 shows the results for the critical CTOD values. Moreover, the plastic component can be quantified throughout the crack mouth opening displacement in Table 2 and can be analyzed in Figure 7, which uses the fractured surface image obtained by SEM fractography showing the crack-tip blunting and brittle fracture region.

\section{Results and Discussion}

According to the procedure shown in Figure 2, the graphics of likelihood patterns are obtained as shown in Figure 8 . The likelihood plot for the stress-only criterion is shown in Figure 8-a, while that for the stress-strain 
Table 2. List of critical CTOD values and plastic component.

\begin{tabular}{|c|c|c|c|}
\hline \multirow[t]{3}{*}{ Specimen No. } & $\begin{array}{l}\text { Load at } \\
\text { fracture }\end{array}$ & $\begin{array}{c}\text { Plastic } \\
\text { component of } \\
\text { crack mouth } \\
\text { opening } \\
\text { displacement }\end{array}$ & $\begin{array}{l}\text { Critical } \\
\text { CTOD }\end{array}$ \\
\hline & $\mathrm{P}$ & $\mathrm{Vp}$ & \\
\hline & $\mathrm{kN}$ & $\mathrm{mm}$ & $\mathrm{mm}$ \\
\hline $\mathrm{z}-10$ & 19.10 & 0.054 & 0.043 \\
\hline $\mathrm{z}-11$ & 18.43 & 0.078 & 0.049 \\
\hline $\mathrm{z}-12$ & 13.04 & 0.005 & 0.015 \\
\hline$z-13$ & 17.86 & 0.227 & 0.087 \\
\hline$z-14$ & 19.13 & 0.787 & 0.238 \\
\hline$z-15$ & 19.12 & 0.010 & 0.029 \\
\hline$z-16$ & 15.78 & 0.004 & 0.019 \\
\hline$z-17$ & 14.28 & 0.013 & 0.020 \\
\hline $\mathrm{z}-18$ & 18.97 & 0.066 & 0.046 \\
\hline$z-19$ & 20.56 & 0.194 & 0.086 \\
\hline $\mathrm{z}-20$ & 12.00 & 0.000 & 0.011 \\
\hline $\mathrm{z}-21$ & 20.58 & 0.223 & 0.093 \\
\hline $\mathrm{z}-22$ & 17.30 & 0.016 & 0.028 \\
\hline$z-23$ & 19.89 & 0.198 & 0.085 \\
\hline$z-24$ & 18.79 & 0.065 & 0.046 \\
\hline $\mathrm{z}-25$ & 20.18 & 0.198 & 0.086 \\
\hline $\mathrm{z}-26$ & 19.70 & 0.317 & 0.117 \\
\hline$z-27$ & 16.92 & 0.019 & 0.028 \\
\hline $\mathrm{z}-28$ & 14.26 & 0.003 & 0.017 \\
\hline$z-29$ & 19.54 & 0.070 & 0.050 \\
\hline$z-30$ & 15.21 & 0.011 & 0.021 \\
\hline$z-31$ & 19.40 & 0.078 & 0.051 \\
\hline$z-32$ & 17.20 & 0.024 & 0.029 \\
\hline$z-33$ & 19.16 & 0.153 & 0.070 \\
\hline$z-34$ & 19.59 & 0.139 & 0.067 \\
\hline$z-35$ & 12.68 & 0.001 & 0.013 \\
\hline$z-36$ & 17.50 & 0.034 & 0.033 \\
\hline$z-37$ & 19.81 & 0.278 & 0.106 \\
\hline$z-38$ & 17.48 & 0.349 & 0.119 \\
\hline
\end{tabular}

criterion is shown in Figure 8-b. Also, the likelihood values for the Weibull stress criterion are shown in Figure 9 Since Equation 10 represents the likelihood function, the highest values printed on the graphs indicate the highest likelihood values obtained for chosen shape and scale parameters.

To summarize, in Figures 8 and 9, there is a wide region having the highest value. Hence it is somewhat difficult to pinpoint the optimum point. In other words, the optimum point to $m$ and $\beta_{\mathrm{f}}$ values are still difficult to be identified even if the stress-strain criterion is applied. Although it is difficult to pinpoint the parameters that lead to the highest likelihood in Figure 8a, the optimum point can be determined as $m=13, \beta_{\mathrm{f}}=5700 \mathrm{MPa}$. Assuming these values, the present model was applied, taking into account the stress-only model, hence the scatter of critical CTOD can be predicted.

Through the experiment data, the statistical distribution of critical CTOD is assumed to obey the Weibull distribution. Figure 10 shows the experimentally determined critical CTOD distribution and regression line (solid line: $m_{\delta}=1.42, b_{\delta}=0.065 \mathrm{~mm}$ A set of the predicted CTOD values was approximated by

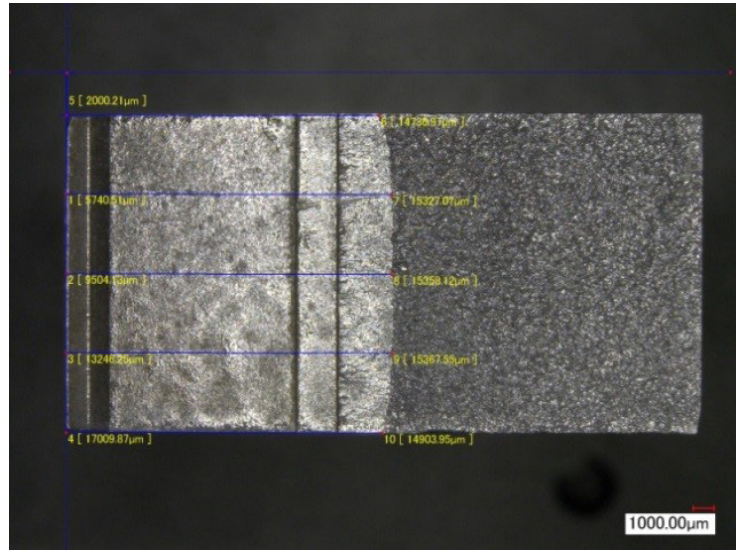

Figure 6. Fatigue crack length measurement.

the Weibull distribution and a dashed line was created in Figure 10 to represent the best-fit prediction by the stress only criterion $\left(m=13, \beta_{f}=5700 \mathrm{MPa}\right)$. Shape and scale parameters of the dashed line were determined as $m_{\delta}=1.39$ and $b_{\delta}=0.040 \mathrm{~mm}$. These values are close to experimentally determined values: as $m_{\delta}=1.42$ and $b_{\delta}=0.065 \mathrm{~mm}$.

Ruggieri ${ }^{9}$ determined the parameters of critical Weibull stress from the statistical distribution of critical CTOD values. He concluded that a single set of critical CTOD values from the same specimen shape cannot determine the Weibull shape and scale parameters, but different specimen shapes with different plastic constraints are necessary to determine the Weibull parameters. The present result has shown the same result, as shown in Figure 9.

The Weibull stress-only criterion is based on the assumption that the so-called "Weibull stress" exists as a material parameter that represents the toughness scatter. And the statistical distribution of critical CTOD value can be predicted from the criterion. This criterion not only predicts the statistical distribution of critical CTOD values but also predicts change of the critical CTOD values with specimen size and shape, i.e. the change in plastic constraint. The essential point in this criterion is that the critical Weibull stress obeys the Weibull statistical distribution.

In the present analysis, the statistical distribution of the local fracture stress was assumed, both in the stress and stress-strain criterion. Both criteria predict the statistical distribution of critical CTOD values from the assumed statistical distribution of the local fracture stress. The present paper has confirmed that the same prediction is derived between the Weibull stress criterion and the local fracture stress criterion (stress-only criterion) if the Weibull statistical distribution is assumed in the latter criterion. In this case, both criteria seem to represent the same physical meaning of brittle fracture. Basically, however, it is not necessary for the local fracture stress (stress only and stress-strain criterion) to assume the Weibull statistical distribution.

It has been claimed as a problem that a unique set of shape and scale parameters of the critical Weibull stress is difficult to be determined from a single set of critical CTOD values. This might indicate that a one-to-one relationship between critical Weibull stress distribution and critical CTOD values does not exist. The same seems true for the local fracture stress 


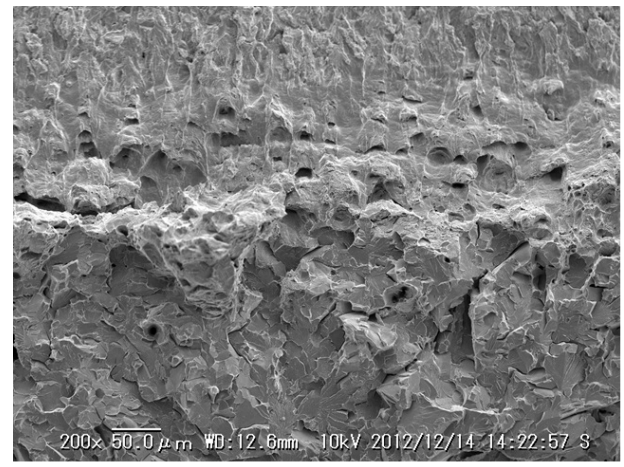

a) Z-14: $\delta c=0.238 \mathrm{~mm}$

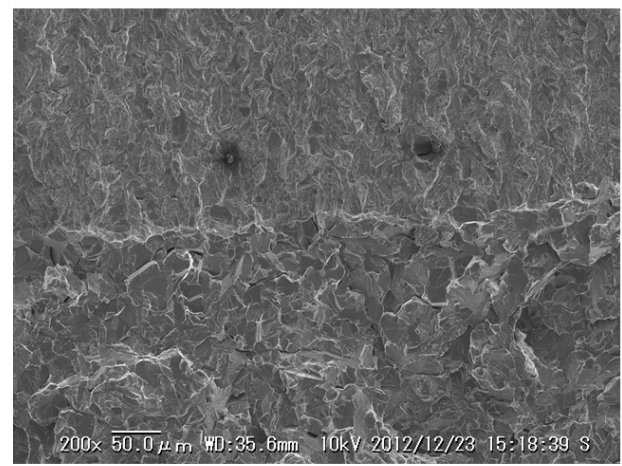

c) Z-22: $\delta \mathrm{c}=0.028 \mathrm{~mm}$

Figure 7. SEM fractography images, showing crack-tip blunting.

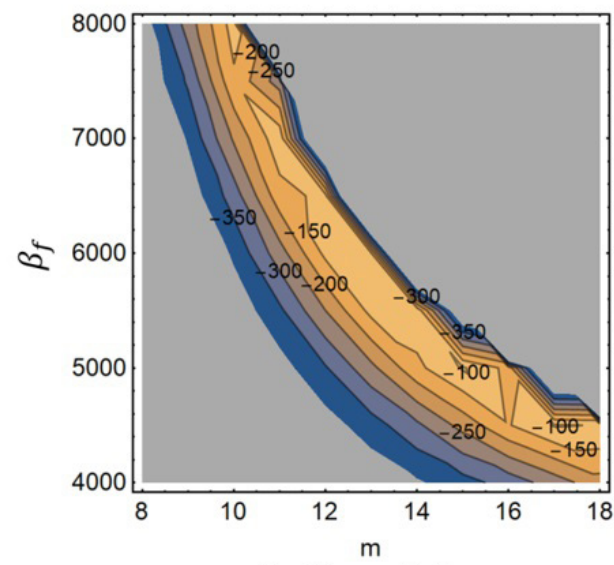

a) Stress criterion

Figure 8. Likelihood pattern for local fracture stress.

criterion (both stress and stress-strain criterion). Although it might look like a lack of the theory, it might be a natural consequence because different microstructure of steels can give the same critical CTOD distribution. If this is true, it might be impossible, in principle, to determine the critical Weibull stress parameters from a set of critical CTOD values. Conversely, however, the statistical distribution of the critical CTOD must be determined uniquely, once the microstructure is fixed. One of the present authors ${ }^{11}$ proposed a method to predict Charpy impact property from microstructural parameters

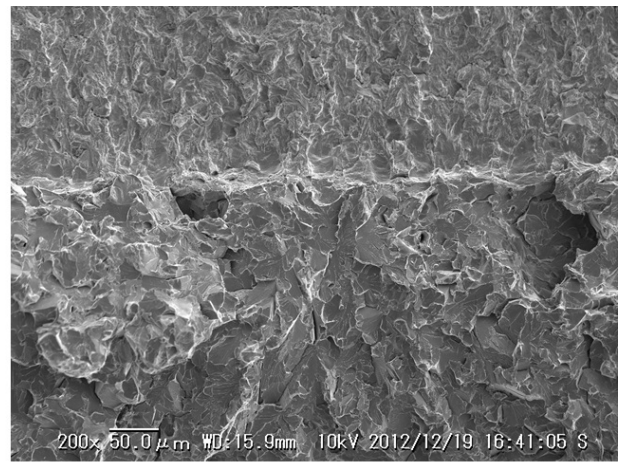

b) Z-19: $\delta \mathrm{c}=0.086 \mathrm{~mm}$

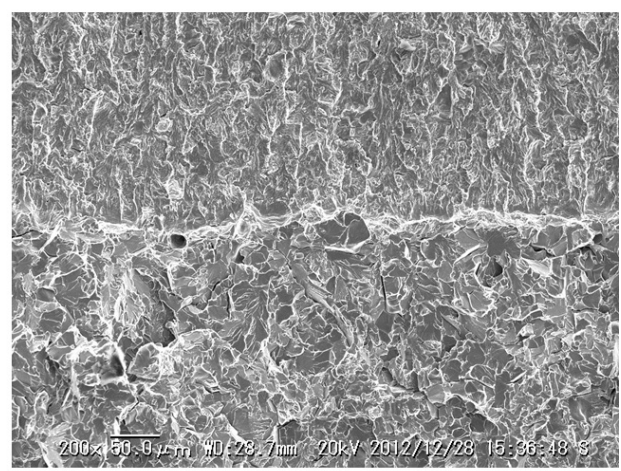

d) Z-31: $\delta \mathrm{c}=0.051 \mathrm{~mm}$

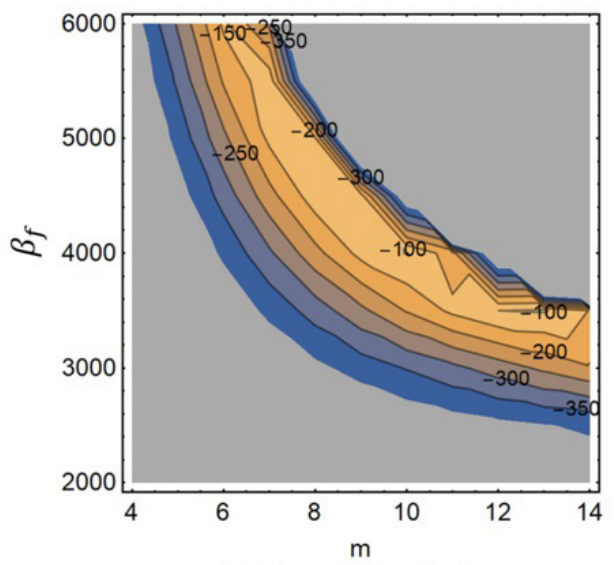

b) Stress-strain criterion

and stress-strain curve. The statistical distribution of the local fracture stress is determined from microstructural parameters (grain size, brittle micro-phase size, etc.) in the model. It does not necessarily obey the Weibull statistical distribution but it is determined from the distributions of these microparameters. The criteria proposed by the present paper (stress only criterion and stress-strain criterion) might be able to apply for predicting the statistical distribution of fracture toughness, like CTOD. 


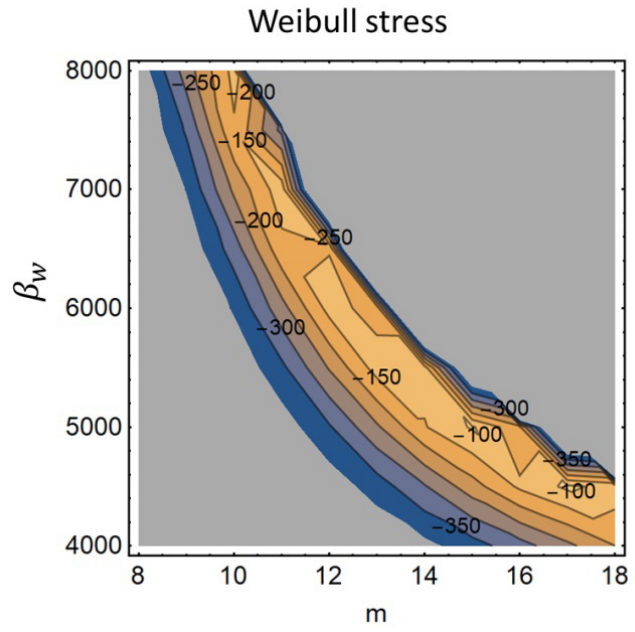

Figure 9. Likelihood pattern for the Weibull distribution.

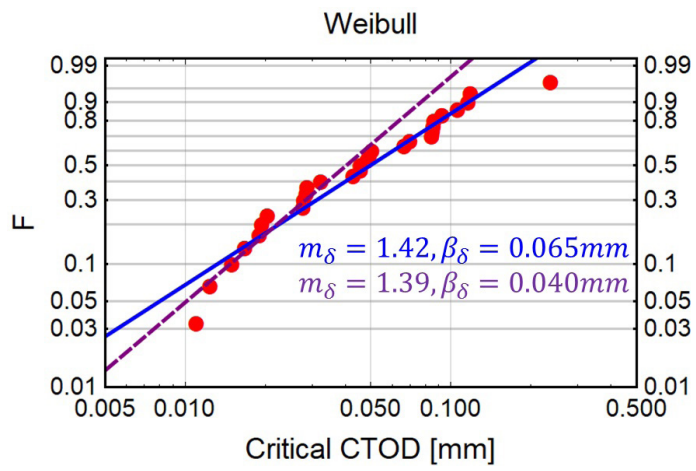

Figure 10. Comparison of the experimental and predicted statistical distribution of critical CTOD.

\section{Conclusions}

The results obtained by the proposed model exemplify the scattering of the material toughness on the presence of cleavage fracture. For this type of fracture, the weakest link theory plays an important role to explain the dispersion of the critical CTOD values in the experiments.

Furthermore, it is possible to mention the following points about the results:

- Through the present model, the likelihood function is used to find optimum values for shape and scale parameters in order to represent the failure probability in the experiments, although, it is difficult to pinpoint these values because there is a number of values,

$\left\{\mathrm{m}, \beta_{\mathrm{f}}\right\}$, that can reproduce the experimental critical CTOD values, even if the probability of crack initiation by strain is introduced.

- This work shows that when the statistical distribution of critical CTOD is used to obtain the shape and scale parameters of the critical Weibull stress, the determination of the parameters is not achieved because of the wide range of the parameter values, as shown in Figure 8, and 9.

- However, when the shape and scale parameters of the critical Weibull stress (or, the statistical distribution of local fracture stress) are set, the statistical distribution of critical CTOD can be properly obtained, since this process corresponds to predicting critical CTOD from microstructure.

- The probability equation line was successfully predicted, despite the proposed model used Equation 2 representing the stress-only criterion model, instead of Equation 3 that may be used for the stress-strain criterion model. Then, the approach provides a computational representation for the experimental results through the Weibull statistical distribution of critical CTOD.

- In Figure 10, the stress-only criterion was applied to plot the best-fit prediction. However, the stressstrain criterion could be also assumed to represent the statistical distribution of critical CTOD values.

The parameters of a predicted line for the failure probability are into the range established by the literature review and their values are not far from the parameter values for the experimental line. However, this work proposes a computational model using the HRR singularity to represent the maximum principal stress of each element near the crack-tip. The use of the finite element method would be also effective to provide a representation for the experiments and portray the strain in the vicinity of the crack.

\section{Acknowledgment}

The author, Freire, R.M.M., thanks CAPES - Coordenação de Aperfeiçoamento de Pessoal de Nível Superior for the financial support through the Science Without Borders program scholarship to conduct studies as a research student at Department of Systems Innovation in the University of Tokyo, Japan, from November 2012 to May 2013. The author, Freire, R.M.M., thanks Professor Shuji Aihara for all his supervision and guidance to conduct studies on fracture mechanics and experimental works at his Laboratory at The University of Tokyo and his support to the present paper's writing. Finally, we thank Ms. Maria Paula Moura Pini for the critical reading of English.

\section{References}

1. Beremin FM, Pineau A, Mudry F, Devaux J-C, D'Escatha Y, Ledermann P. A local criterion for cleavage fracture of a nuclear pressure vessel steel. Metall Trans, A, Phys Metall Mater Sci. 1983;14(11):2277-87.

2. Bordet SR, Tanguy B, Besson J, Bugat S, Moinereau D, Pineau A. Cleavage fracture of RPV steel following warm pre stressing: micromechanical analysis and interpretation through a new model. Fatigue Fract Eng Mater Struct. 2006;29(9-10):799-816.

3. Anderson TL. Fracture mechanics: fundamentals and applications. 3rd ed. Boca Raton: CRC Press; 2005.

4. Ruggieri C, Minami F, Toyoda M, Hagiwara Y, Inoue T. Local approach to notch depth dependence of CTOD results. J Soc Nav Archit Jpn. 1992;1992(171):493-9.

5. Tagawa T, Kayamori Y, Hira H. Statistical scatter of fracture toughness in the ductile-brittle transition of a structural steel. Eng Fract Mech. 2010;77(16):3077-86.

6. Kawata I, Yoshizu S, Nakai H, Shibanuma K, Aihara S. Development of new formulation and likelihood function on probabilistic fracture initiation model. Procedia Struct Integrity. 2016;2:2463-70. 
7. Lin T, Evans AG, Ritchie RO. A statistical model of brittle fracture by transgranular cleavage. J Mech Phys Solids. 1986;34(5):477-97.

8. Bertolo VM, Jiang Q, Walters CL, Popovich VA. Effect of microstructure on cleavage fracture of thick-section quenched and tempered S690 High-Strength steel. In: Li J, Zhang M, Li B, Monteiro SN, Ikhmayies SS, Kalay YE, et al., editors. Characterization of minerals, metals, and materials. Cham: Springer; 2020. p. 155-68.

9. Ruggieri C. A probabilistic model including constraint and plastic strain effects for fracture toughness predictions in a pressure vessel steel. Int J Press Vessels Piping. 2016;148:9-25.

10. Sih GC. Probabilistic fracture mechanics and reliability. Cham: Springer Science \& Business Media; 2013.

11. Kunigita M, Aihara S, Kawabata T, Kasuya T, Okazaki Y, Inomoto M. Prediction of Charpy impact toughness of steel weld heat-affected zones by combined micromechanics and stochastic fracture model-Part I: model presentation. Eng Fract Mech. 2020;230:106965.
12. Gao X, Ruggieri C, Dodds RH Jr. Calibration of Weibull stress parameters using fracture toughness data. Int J Fract. 1998;92(2):175-200.

13. Rice J, Rosengren GF. Plane strain deformation near a crack tip in a power-law hardening material. J Mech Phys Solids. 1968;16(1):1-12.

14. Hutchinson JW. Crack-tip singularity fields in nonlinear fracture mechanics: a survey of current status. In: 5th International Conference on Fracture; 1981 Mar-Apr 29-3; Cannes, France. Proceedings. Oxford: Pergamon Press; 1981. p. 2669-84.

15. Gurland J. Observations on the fracture of cementite particles in a spharoidized $1.05 \%$ steel deformed at room temperature. Acta Metall. 1972;20(5):735-41.

16. Minami F, Brückner-Foit A, Munz D, Trolldenier B. Estimation procedure for the Weibull parameters used in the local approach. Int J Fract. 1992;54:197-210.

17. The Japan Welding Engineering Society. WES1108: standard test method for crack-tip opening displacement (CTOD) fracture toughness measurement. Tokyo: JWES; 1995. 